\title{
IJCAI 2018 - Chinese Dominance Established
}

\author{
Matjaž Gams \\ Jožef Stefan Institute, Jamova 39, 1000 Ljubljana, Slovenia \\ E-mail:matjaz.gams@ijs.si
}

\section{Editorial}

In July 2018 in Stockholm, ICML, AAMAS, ICCBR and SoCS joined with IJCAI and ECAI to establish the first major worldwide AI event. This paper is about the resulting IJCAI-ECCAI event [1].

The 27th International Joint Conference on Artificial Intelligence and the 23rd European Conference on Artificial Intelligence merged with the other events to form a single conference. Around 7000 participants divided their time between these conferences over 14 days as one fee covered the entrance to all the events. As a consequence, several researchers attended several conferences, which in itself was a major achievement. Namely, the conferences and even the individual sections of the conferences are becoming so specialized that AI researchers are becoming oblivious to the achievements being made in a related area, leading to specialization and small incremental improvements, thereby deterring major innovations. Fortunately, in 2018 there was a serious attempt to reintegrate the field.

For the organizers, the super-event joint conferences represented a huge effort, but everything ran smoothly - albeit with a couple of small exceptions, as usual. One of them was the initial robot dance, where a Nao robot performed a predefined sequence of moves, which the human dancer enriched with dynamics and scope. The glitch was a loss of sound during the event (deliberate or by accident?). Added to this, the lack of any AI in the performance was a huge issue for many of the participants; in particular, the absence of true AI, one of the central themes of the conference. However, the artistic impression was there. Perhaps not surprisingly, as the small Nao robot was clearly physically and dynamically very much inferior to the flexible human dancer, a kind of reverse of David and Goliath seemed to be taking place. Also, the big 1000+ lecture rooms were organized in such a way that at no time was everybody sitting down, instead there seemed to be 5-10 people in motion at any moment. That aside, Stockholm is a traditional, open, metropolitan city that has hosted conferences for up to several tens of thousands of participants before, and the AI organizers have extensive experience as well; so by any measure the event must be considered as an organizational success.

The IJCAI-ECCAI joint event involved a record 3470 submitted papers: $37 \%$ more than in 2017, while the 2017 event was $11 \%$ up on the previous year, confirming the steady growth from 2007 on. AI continues to progress as a scientific field and as an area of human interest.

The first major technical impression in 2018 was that Chinese dominance has finally been established. Eclatantly! In 2017, 37\% of the papers were Chinese, while a year later this figure was $46 \%$. Only $9 \%$ increase, one might say, but the 2017 conference was in Australia, with strong Chinese ties, while Stockholm is in Europe, and it was a joint European and international IJCAI conference, meaning around half of the event was basically a European conference. Despite that, European and American papers constituted around $20 \%$ each, while several authors, in particular from the USA, were also Chinese. Astonishment and admiration are the right words to describe this Chinese success.

The more detailed numbers are as follows: from the 710 accepted papers (21\% acceptance rate), 325 came from China, 129 from EU (UK 37, France 22, Italy 18, Germany 15, Austria 12), USA 122, Singapore 26, Australia 23, Japan 17, Israel 13, etc.

When asked if it is reasonable to limit nonEuropean papers at least for the ECCAI conference, say to $50 \%$, several of the researchers expressed concern that it would mean that several of the best non-European papers would then end up being presented at other conferences. Several of the Chinese papers were indeed of high quality, demonstrating Chinese innovation, good education and the major support for AI in China. There were some concerns that the Chinese papers often follow a pattern with a specific idea, lots of complicated mathematics and an unverified empirical comparison. But that is true for many other papers as well. It should be noted, in addition, that due to several national European research policies, it is often nearly worthless for domestic evaluations to publish a paper at IJCAI or ECAI, since all that counts for these researchers are journal publications. The absence of more high-quality 
European papers might therefore be partially attributed to the strange European scientific policies. Some of these issues were discussed at the panels, as presented in Figures 1 and 2.

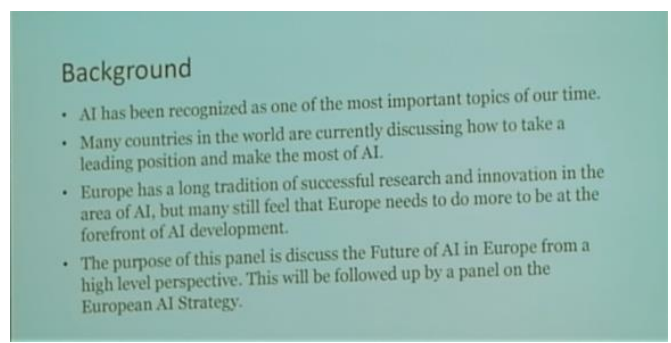

Figure 1: At a panel about European AI, the importance of the field for European progress was clearly established.

In some presentation, e.g., the one shown in Figure 1, the European position and the selfevaluation were not exactly in accordance with the percentage of conference papers. Some other positions even sounded a bit like a clip from a galaxy far, far away. But in reality, the panels were of high quality and several essential issues and initiatives were raised. Several panelists mentioned that there is no AI coordination in Europe, even though the EU is still the no. 1 world economy. In terms of AI funding, the USA prevails over China $2: 1$, and China prevails over the EU, again by $2: 1$. Such estimates might be misleading since the nominal comparison took place - instead, real economy (how many kilograms of sugar or of steel) already puts China above USA in terms of scientific funding.

There are two important differences between the USA and the EU: the USA executes bold international policies, whereas the EU finds its soft approach is sometimes hurting its economy and society. The EU used to be no.1 in computer science; now it is no. 3. Lots of this falling behind was not necessary at all; instead there are subjective leadership reasons for the decline, e.g., the EU patent system is enormously complicated and bureaucratic compared to the American one. Another problem: the UK has the best European AI based on many criteria, and so Brexit will make this situation worse for the EU. Whereas top EU projects like H2020 represent world-class research, and the EU is still leading in many areas of business and science, the strong scientific funding for key areas as well as policies to support them are lacking.

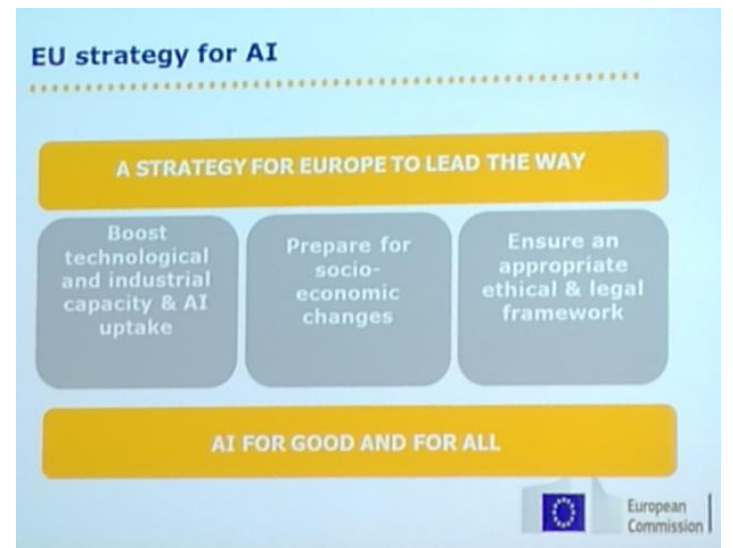

Figure 2: EU strategy involves three elements: science/technology, socio-economic changes and the social framework.

While the EU is as concerned with legal issues as it is with the research, China has significantly improved its AI efforts through governmental and private funding, and there is no major rift in the government. The democracies of several European countries and the USA are torn apart because of ideological and political antagonisms, instead of focusing on technical progress. For Chinese researchers, the road to success and obtaining a good position at home is to publish at major AI conferences, in major journals and join established researcher teams in the USA or Europe. For Europeans, it is possible to follow the Chinese path, but no European country offers a several-timeshigher salary for researchers returning home, like China does. While the presidents of superpowers from the USA to Russia declare the tremendous importance of AI in relation to world dominance, the percentage of papers best demonstrates who supports the field the most. This is not to say that all major countries are not increasing their AI funds significantly. For example, the EU has presented its plans at IJCAI (Figure 3): first, a $70 \%$ increase, followed by a $100 \%$ and then another $100 \%$. The US Department of Defense (DoD) established the Joint AI Center (JAIC). It will host the DoD's 600 AI projects with an estimated $\$ 1.7$ billion over 6 years. As predicted, AI will likely change the nature of welfare, along with several other fields. However, without sufficient AI research, nobody can expect to maintain its leading position in the world. 


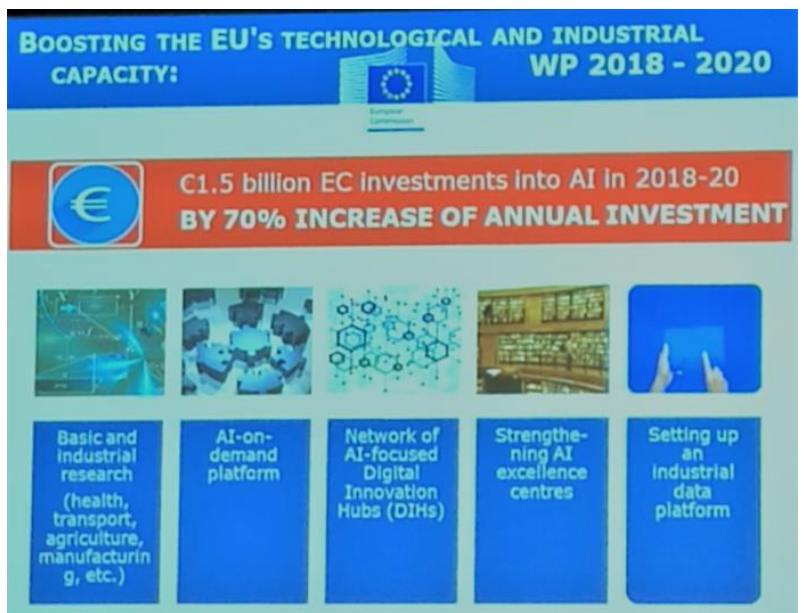

Figure 3: The EU will significantly increase AI funding. Finally. Will national governments follow?

A closer look at the EU plan reveals that there are several new ideas, as presented in Figure 4. Among others, the EU will fund the open AI platform, which is at least partially influenced by Elon Musk's, which by the way won the first 5 vs 5 Dota2 game with expert players (some small additional limitations). The EU plan was probably the major AI strategy presented at the conference. While China does it on its own and the USA allocates most funds to military applications, the EU is focused on a public, general, AI-boosting plan to benefit everybody. That is for sure great news, not only for AI in Europe, but for humanity as a whole.

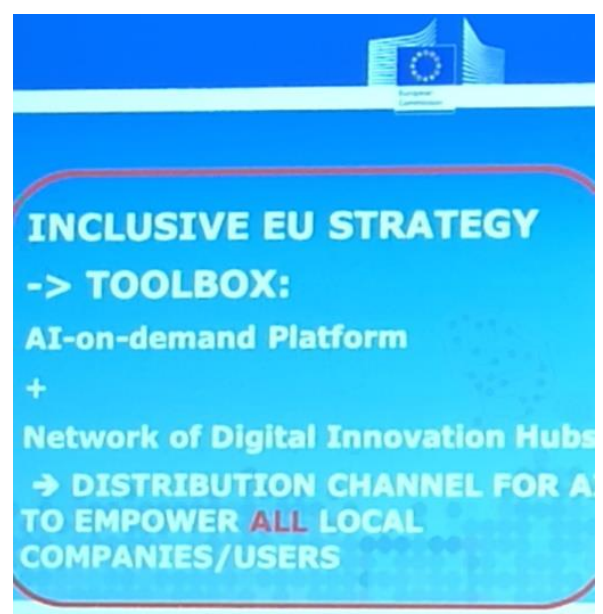

Figure 4: The EU strategy introduces several integrating $\mathrm{EU}$ components, including the $\mathrm{AI}$ toolbox and the Network of Digital Innovation Hubs. Unfortunately, many of the most advanced AI hubs are in the UK.

Several new mechanisms like CLAIRE are already active (https://claire-ai.org/): "an initiative by the European AI community that seeks to strengthen European excellence in AI research and innovation."

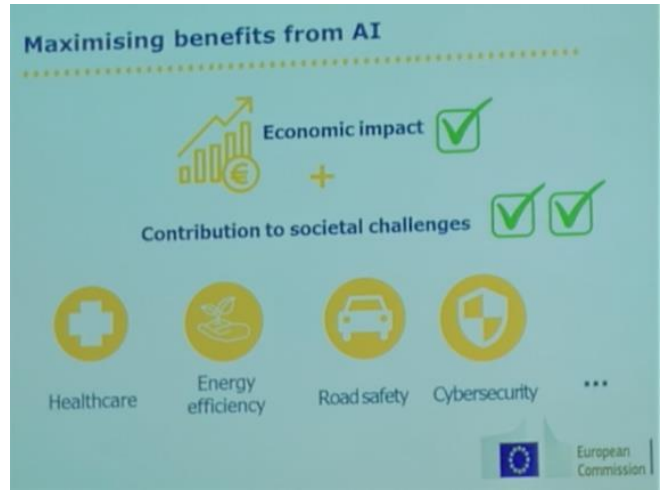

Figure 5: The EU expects that AI progress will bring several benefits, from the economic impacts to solutions to the societal challenges. There are several areas that will see major advances in the near future, such as healthcare.

"If Europe were to fall behind in AI technology, we would be likely to face challenging economic consequences, an academic brain drain, reduced transparency, and increasing dependency on foreign technologies, products and values. The CLAIRE initiative presents a proposal to avoid that."

"The CLAIRE initiative aims to establish a panEuropean network of Centres of Excellence in AI, strategically located throughout Europe, and a new, central facility with a state-of-the-art, "Googlescale", CERN-like infrastructure - the CLAIRE $\mathrm{Hub}$ - that will promote new and existing talent and provide a focal point for the exchange and interaction of researchers at all stages of their careers, across all areas of AI. The CLAIRE Hub will not be an elitist AI institute with a permanent scientific staff, but an environment where Europe's brightest minds in AI meet and work for limited periods of time. This will increase the flow of knowledge among European researchers and back to their home institutions."

Maybe, we should also remember the times when science was not a business, when we researched not for the purpose of cash, but for reasons of fundamental curiosity, a desire to improve our knowledge. Some spirit of that kind is still observable at the conferences and was also demonstrated, for example, by the computer chess tournament. During the breaks many participants occasionally stopped by and observed the most interesting matches. Komodo won the World Computer Chess Championship 2018 after a playoff with GridGinkgo. In third place was Jonny, due to a win over Leela Chess Zero. The latter was observed with much interest, due to having implemented AlphaZero for the PC. It was not a match for the best programs, instead it played out 
very differently - intuitively, lucidly and errorprone. Obviously, it lacked the power of the Google computers to validate its fancy ideas, often in the form of sacrifices. Figure 6 shows the Komodo team, who received the Shannon Trophy (and replica) from the chairman of the ICGA David Levy.

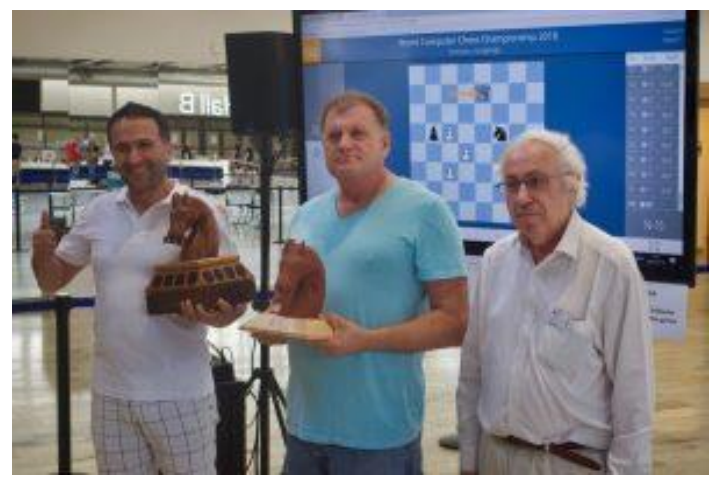

Figure 6: Komodo was again the computer chess champion on PCs. Leela Chess Zero, a PC version of the Alpha Zero, played lucidly, but had no chance against the hard logic of Komodo.

In Stockholm 2018 the social meetings of societies were boosting the exchange of information and cooperation, be it inside the EU or international societies. For example, the EurAI meeting (Figure 7), IJCAI AI societies meeting, IFIP meetings, etc.

The IJCAI report should first of all be about scientific achievements. In 2018 there was distinct, albeit rather expected, progress. Indeed, there were plenty of reasonably novel improvements, and indeed the major theme was a challenging one: How to grow a mind, a true AI - but that was it. Quite enough for many, but a bit too classical for others. Furthermore, the AI influence on our everyday life has already achieved much greater impact than generally anticipated in the public opinion: every day AI makes around 100 trillion decisions, meaning it is thoroughly embedded into our society. Coupled with other ICT achievements, human society long ago developed into an information society - an integration of humans and ICT systems, and an integration of human society and technology. This is one of the reasons why nobody understands what is actually going on - social scientists understand society, while engineers and technical scientists understand technological systems, and finally nobody understand the two embedded and integrated into one unity - kind of Borg stuff, just that the unifying essence is the web and ICT and AI services. Another analogy is related to computer chess - when humans play based on their own brains, the inferiority and inability to understand complex relations are evident. Only coupled with powerful computers and advanced AI programs can we hope to decipher the societal changes and trends, and propose good solutions.

With regards to the novel applications, Tambe's group stood out from many - their security AI designing daily schedules for airports, harbors and other relevant facilities is employed at several locations worldwide. It has even been given to 60 wildlife parks to cope better with poachers. That is one of the successful applications, accompanied by a huge mass of new research systems, e.g., a novel HW and SW embedded system connected to the patient's spine that enables a paralyzed patient to stand up.

New classes of applications are emerging, e.g., in visual tasks. DNNs can transform a human face into another, even create a new face never seen before. An animal, say a horse, can be camouflaged into zebra stripes and it can move freely around in a simulated video. Systems speak perfectly and listen better than humans; they can sharpen a picture or translate from voice online. Google search is using DNNs to capture the best answer to a question.

On the other hand, there are seemingly bizarre simple problems that researchers have a hard time dealing with. While it is generally accepted that DNNs outperform humans in visual classification tasks, it is still a big problem to transfer one ML system based on examples from a specific hospital and specific scanning devices to another. The technical differences are small, causing human experts no problem, but for the DNNs these small details significantly impair the quality. Until recently, that is. At the conference, several solutions related to transfer learning, general AI and also real AI were presented and discussed. Why should AI systems not learn like children, gathering knowledge and learning from there on with a small number of examples, even a single one? 


\section{Babies learn how the world works by observation}

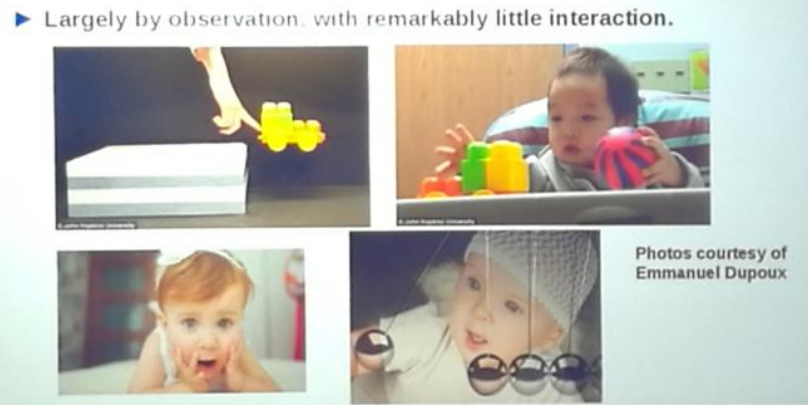

Figure 7: Children learn in a very different way to AI. Why not copy them?

There is shallow, i.e., current AI, deep AI (also claimed as shallow AI), real AI, and fake AI. The last one refers to chatbots, i.e., virtual assistants, where human operators often jump in communications and leave users under the impression that it is AI on its own. The real AI was one of the major themes of the conference, which is quite a big difference from the previous conferences, where the primary goal was to complete the tasks better than expert humans, be it chess or detecting malignant tissue patterns. Now the task is different - perform at the level of children aged a couple of years. While supervised learning clearly achieves top performance, compared to humans it needs far too many examples, which is not acceptable, at least for the slow humans. Similarly, reinforcement learning needs way too many trials. Furthermore, machines do not have common sense compared even to young children.

In terms of the ban on autonomous weapons, more and more societies and countries are joining the ban. EurAI, as the union of all AI European societies and the second largest AI society in the world, also supports the ban.

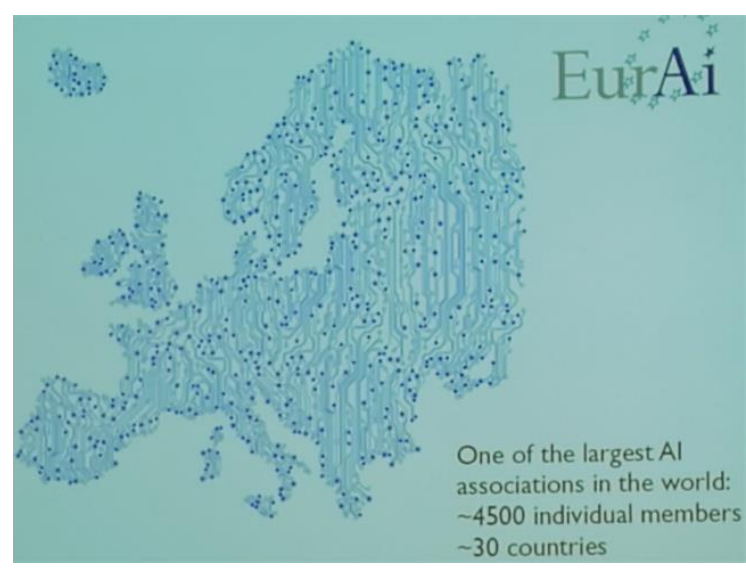

Figure 7: More and more societies and countries are joining the ban on autonomous weapons. EurAI, as the union of all AI European societies, also joined the efforts.

In 2018, the debate on banning autonomous weapons was held in the UN and in the European Parliament:

https://www.stopkillerrobots.org/2018/07/parliamen ts-2/. The list of institutions supporting the ban is here: https://www.stopkillerrobots.org/coalition/.

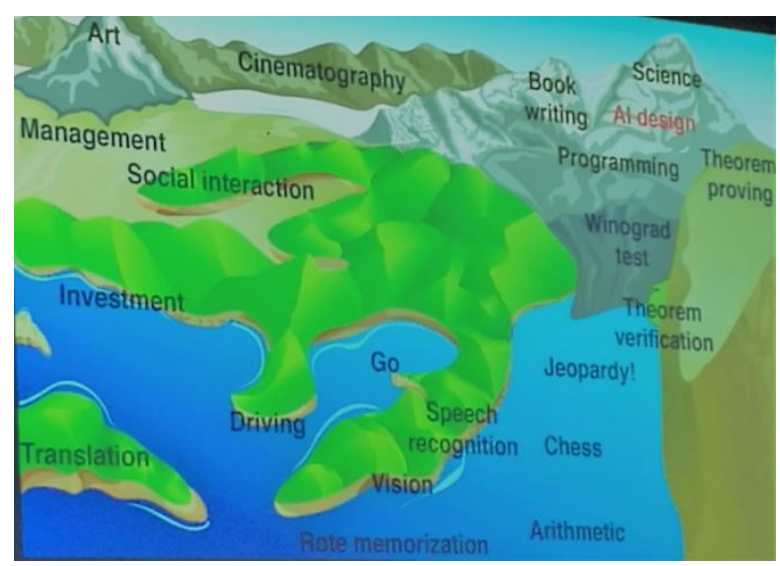

Figure 8: Tegmark's view of the AI field.

Another interesting approach is to generate general AI, as was the case in the 2018 IJCAI conference. Currently, the majority opinion among AI researchers is that general AI is possibly 10 years away. It is probably not that the AI community lacks computer power or finances; it is the novel ideas that we are striving for. There is also a reasonable consensus that AI can, could, should and will help humans solve major human societal problems. Scientists should avoid the politics, especially the discrepancies between different ideological or political tracks, and defer from attacking colleagues along these lines. Science should be kept as separate as possible from politics and ideology. With these words from Tegmark (Figure 8) we look into a bright EU AI, AI and human future.

\section{References}

[1] IJCAI 2018 conference (https://www.ijcai-18.org/). 
